\title{
LOWER BOUNDS ON THE PERFORMANCE OF THE MAP EQUALIZER WITH $A$ PRIORI OVER MIMO SYSTEMS
}

\author{
Chaabouni Sihem
}

\author{
ISECS, Route Menzel Chaker km 0.5 \\ BP 868, 3018 Sfax, Tunisia \\ chaabouni_sihem@yahoo.fr
}

\author{
Noura Sellami \\ ISECS, Route Menzel Chaker km 0.5 \\ BP 868, 3018 Sfax, Tunisia \\ sellami_noura@yahoo.fr
}

Aline Roumy

IRISA-INRIA, Campus de Beaulieu 35042 Rennes Cedex, France aline.roumy@irisa.fr

\begin{abstract}
In this paper, we consider a transmission of Quadrature Phase Shift Keying (QPSK) symbols over a frequency selective Multiple Input Multiple Output (MIMO) channel. We propose to study analytically the impact of a priori information (provided for instance by a decoder in a turbo equalizer) on the maximum a posteriori (MAP) equalizer performance. We derive analytical expressions of tight lower bounds on the bit error probability at the output of the equalizer. Simulations show that the analytical expressions approximate well the bit error rate (BER) at the output of the MAP equalizer.
\end{abstract}

\section{INTRODUCTION}

The high data rate communication systems are impaired by intersymbol interference (ISI). To combat the effects of ISI, an equalizer has to be used. The optimal soft-input soft-output equalizer, in the sense of minimum bit error rate (BER), is based on the maximum a posteriori (MAP) criterion. In this paper, we consider the case where the MAP equalizer has a priori information on the data. The a priori information are provided by another module in the receiver, for instance a decoder in a turbo-equalizer [1]. In a turbo-equalizer, the equalizer and the decoder exchange extrinsic information and use them as a priori in order to improve their performance.

In [2], the authors studied analytically the impact of a priori information on the MAP equalizer performance in the case of Binary Phase Shift Keying (BPSK) modulation for single input single output (SISO) systems. The aim of our paper is to generalize the study to the case of Multiple Input Multiple Output (MIMO) systems with Quadrature Phase Shift Keying (QPSK) modulation. To do this, we derive analytical expressions of tight lower bounds on the MAP equalizer BER performance. Simulations show that these expressions approximate well the BER at the output of the MAP equalizer.

This paper is organized as follows. In section 2 , we give the system model. In section 3, we derive analytical expressions of tight lower bounds on the BER at the output of the MAP equalizer. In section 4 , we give simulation results.

Throughout this paper matrices are upper case and vectors are underlined lower case. The operator $(.)^{T}$ denotes transposition, the operator $(.)^{H}$ denotes Hermitian and $I_{N}$ represents the $N \times N$ identity matrix.

\section{SYSTEM MODEL}

We consider a data transmission system over a frequency selective MIMO channel with $P$ transmit antennas and $N$ receive antennas. We assume that transmissions are organized into bursts of $T$ symbols per transmit antenna and the channel is invariant during one burst. The channel between each transmit antenna and each receive antenna has a memory of $L$. The input information bit sequence $\underline{b}=\left(b_{2(1-L)}^{(1)}, \ldots, b_{(2(T-1)+1)}^{(1)}, b_{2(1-L)}^{(2)}, \ldots\right.$,
$\left.b_{(2(T-1)+1)}^{(2)}, \ldots, b_{2(1-L)}^{(P)}, \ldots, b_{(2(T-1)+1)}^{(P)}\right)^{T}$, is mapped to a sequence of QPSK symbols with Gray mapping from the alphabet $A=\{-1-j ;-1+j ;+1-j ;+1+j\}$. The baseband signal received by the $n^{\text {th }}$ antenna sampled at the symbol rate at time $k$ is:

$$
y_{k}^{(n)}=\sum_{p=1}^{P} \sum_{i=0}^{L-1} h_{i}^{(p, n)} x_{k-i}^{(p)}+q_{k}^{(n)},
$$

where $h_{i}^{(p, n)}$, represents the $i^{t h}$ channel tap between the $p^{t h}$ transmit antenna and the $n^{\text {th }}$ receive antenna, for $1 \leq p \leq P$ and $1 \leq n \leq N, x_{k}^{(p)}$, for $1-L \leq k \leq T-1$, are the symbols transmitted by the $p^{t h}$ transmit antenna, $q_{k}^{(n)}$ are modeled as independent random variables of a complex white Gaussian noise with zero mean and variance $\sigma^{2}$, with normal probability density function (pdf) $N_{\mathbb{C}}\left(0, \sigma^{2}\right)$. The equation (1) can be rewritten as follows:

$$
\underline{y}=H \underline{x}+\underline{q},
$$

where $\quad \underline{x}=\left(x_{1-L}^{(1)}, \ldots, x_{T-1}^{(1)}, x_{1-L}^{(2)}, \ldots, x_{T-1}^{(2)}, \ldots, x_{1-L}^{(P)}, \ldots\right.$, $\left.x_{T-1}^{(P)}\right)^{H}$ is the vector of symbols transmitted by all transmit antennas, $\underline{q}=\left(q_{0}^{(1)}, \ldots, q_{T-1}^{(1)}, q_{0}^{(2)}, \ldots, q_{T-1}^{(2)}, \ldots, q_{0}^{(N)}, \ldots, q_{T-1}^{(N)}\right)^{H}$ is the complex Gaussian noise vector, $\underline{y}=\left(y_{0}^{(1)}, \ldots, y_{T-1}^{(1)}, y_{0}^{(2)}\right.$, $\left.\ldots, y_{T-1}^{(2)}, \ldots, y_{0}^{(N)}, \ldots, y_{T-1}^{(N)}\right)^{H}$ is the output vector received by the receive antennas and $H$ is a $N T \times P(T+L-1)$ matrix defined as:

$$
H=\left[\begin{array}{cccc}
H^{(1,1)} & H^{(2,1)} & \ldots & H^{(P, 1)} \\
H^{(1,2)} & \cdots & & H^{(P, 2)} \\
\vdots & & & \vdots \\
H^{(1, N)} & H^{(2, N)} & \cdots & H^{(P, N)}
\end{array}\right]
$$

where $H^{(p, n)}$, for $1 \leq p \leq P$ and $1 \leq n \leq N$, is the channel matrix between the $p^{\text {th }}$ transmit antenna and the $n^{\text {th }}$ receive antenna, which is a $T \times(T+L-1)$ Toeplitz matrix having the following expression:

$$
H^{(p, n)}=\left[\begin{array}{cccccc}
h_{L-1}^{(p, n)} & \ldots & h_{0}^{(p, n)} & 0 & \ldots & 0 \\
0 & h_{L-1}^{(p, n)} & \ldots & h_{0}^{(p, n)} & \ddots & \vdots \\
\vdots & \ddots & \ddots & & \ddots & 0 \\
0 & \ldots & 0 & h_{L-1}^{(p, n)} & \ldots & h_{0}^{(p, n)}
\end{array}\right] .
$$

We note that all channels are normalized: $\sum_{i=0}^{L-1}\left|h_{i}^{(p, n)}\right|^{2}=1$, for $1 \leq p \leq P$ and $1 \leq n \leq N$.

First, we consider the case where no a priori information is provided to the equalizer. The data estimate according to the MAP sequence criterion (or to the Maximum Likelihood (ML) criterion, since there is no a priori) is given by :

$$
\underline{\widehat{x}}=\arg \min _{\underline{u}}\left(\|\underline{y}-H \underline{u}\|: \underline{u} \in A^{P(T+L-1)}\right) .
$$


Let $\underline{x}^{(p)}=\left(x_{1-L}^{(p)}, \ldots, x_{T-1}^{(p)}\right)^{H}$ and $\underline{\widehat{x}}^{(p)}=\left(\widehat{x}_{1-L}^{(p)}, \ldots, \widehat{x}_{T-1}^{(p)}\right)^{H}$ be respectively the subvectors of $\underline{x}$ and $\underline{x}$ corresponding to the $p^{\text {th }}$ transmit antenna. For each transmit antenna $p$, we suppose that there exists an interval of size $m_{p}$ such that all the symbols of $\underline{\widehat{x}}^{(p)}$ are different from the corresponding symbols of $\underline{x}^{(p)}$ while the preceding symbol and the following one are the same. Let $\underline{e}_{m_{p}}=\underline{\widehat{x}}_{m_{p}}-\underline{x}_{m_{p}}$ be the subvector of errors corresponding to this interval, where $\underline{x}_{m_{p}}$ and $\underline{\widehat{x}}_{m_{p}}$ are respectively the corresponding subvectors of $\underline{x}^{(p)}$ and $\underline{\widehat{x}}^{(p)}$. A subevent $\xi_{m}$ of the error event is that " $\underline{\widehat{x}}_{m}=\left(\underline{\widehat{x}}_{m_{1}}, \underline{\widehat{x}}_{m_{2}}, \ldots, \underline{\widehat{x}}_{m_{P}}\right)^{H}$ is better than $\underline{x}_{m}=\left(\underline{x}_{m_{1}}, \underline{x}_{m_{2}}, \ldots, \underline{x}_{m_{P}}\right)^{H}$ " in the sense of the ML metric:

$$
\xi_{m}:\left\|\underline{y}_{m}-H_{m} \underline{\widehat{x}}_{m}\right\| \leq\left\|\underline{y}_{m}-H_{m} \underline{x}_{m}\right\|,
$$

where $m=\sum_{p=1}^{P} m_{p}, \underline{y}_{m}$ and $H_{m}$ are respectively the subvector of $y$ and the subblock of $H$ corresponding to the error interval. We consider the case where the receiver has side information (from a genie) that one of the two sequences $\underline{x}_{m}$ or $\underline{\widehat{x}}_{m}$ was transmitted. When the genie aided equalizer is provided with a priori information, the pairwise error probability that it chooses $\underline{\underline{x}}_{m}$ instead of $\underline{x}_{m}$ is given by [3]:

$$
P_{\underline{x}_{m}, \underline{\widehat{x}}_{m}}=Q\left(\frac{\left\|\underline{\varepsilon}_{m}\right\|}{2 \sigma}\right),
$$

where $\underline{\varepsilon}_{m}=H_{m} \underline{e}_{m}, \underline{e}_{m}$ is the $(m+P(L-1)) \times 1$ error vector and $Q(\alpha)=\frac{1}{\sqrt{2 \pi}} \int_{\alpha}^{\infty} \exp \left(-\frac{y^{2}}{2}\right) d y$.

Let $E_{\underline{e}_{m}}$ be the set of all $\underline{e}_{m}$ achieving the minimum value of $\left\|\underline{\varepsilon}_{m}\right\|$. Let $\pi_{\underline{e}_{m}}$ be the probability that the input sequence $\underline{x}_{m}$ will be such that $\underline{\underline{x}}_{m}=\underline{x}_{m}+\underline{e}_{m}$ is an allowable input sequence for some $\underline{e}_{m}$ in $E_{\underline{e}_{m}}$. Then, the probability that the genie aided equalizer chooses an allowable sequence $\widehat{\underline{x}}_{m}$ instead of $\underline{x}_{m}$ is $\pi_{\underline{e}_{m}} Q\left(\frac{\underline{\underline{m}}_{m}\left\|\underline{\varepsilon}_{m}\right\|}{2 \sigma}\right)$. When the equalizer has no side information, the probability of an error event of length $m$ is lower bounded by the probability achieved by the genie aided equalizer:

$$
P\left(\xi_{m}\right) \geq \pi_{\underline{e}_{m}} Q\left(\frac{\min _{m}\left\|\underline{\varepsilon}_{m}\right\|}{2 \sigma}\right) .
$$

Let $l_{\underline{e}_{m}}$ be the length of the error sequence. The overall probability of error $P_{e}$ is lower bounded by:

$$
P_{e} \geq l_{\underline{e}_{m}} \pi_{\underline{e}_{m}} Q\left(\frac{\underline{\min }_{m}\left\|\underline{\varepsilon}_{m}\right\|}{2 \sigma}\right) .
$$

Our goal is to find analytical expressions of tight lower bounds on the BER at the output of the MAP equalizer with a priori information.

\section{LOWER BOUNDS ON THE BER}

In this section, we propose to derive the expressions of tight lower bounds on the BER at the output of the MAP equalizer with the $a$ priori information for a MIMO system with QPSK modulation. We consider here QPSK modulation with Gray mapping. We choose this mapping since it leads to a linear and simple relationship between bits and symbols, which will be used to calculate the lower bounds. The relationship between BPSK bits $\left(b_{2 k}^{(p)}, b_{2 k+1}^{(p)}\right)$ and QPSK symbols $x_{k}^{(p)}$ is the following:

$$
x_{k}^{(p)}=\left[\begin{array}{ll}
1 & j
\end{array}\right]\left[\begin{array}{c}
b_{2 k}^{(p)} \\
b_{2 k+1}^{(p)}
\end{array}\right] .
$$

The study will be done here for the equalizer using the MAP sequence criterion. It holds for the MAP symbol equalizer using the BCJR algorithm [4] since the two equalizers have almost the same performance as shown in [5, page 814]We assume that channels are perfectly known at the receiver. Moreover, we suppose that a priori observations at the input of the equalizer are modeled as outputs of an additive white Gaussian noise (AWGN) channel. This assumption is generally considered in the analyses of iterative receivers [6]. These a priori observations on bits $b_{j}^{(p)}$, for $2(1-L) \leq j \leq(2(T-1)+1)$ and $1 \leq p \leq P$, are:

$$
z_{j}^{(p)}=b_{j}^{(p)}+w_{j}^{(p)}
$$

where $w_{j}^{(p)}$ are independent random variables of a real white Gaussian noise with zero mean and variance $\frac{\sigma_{a}^{2}}{2}$ with normal pdf $N(0$, $\frac{\sigma_{a}^{2}}{2}$ ). Then, the a priori Log Likelihood Ratios (LLRs) are:

$$
\operatorname{LLR}\left(b_{j}^{(p)}\right)=\operatorname{Ln} \frac{P\left(z_{j}^{(p)} / b_{j}^{(p)}=1\right)}{P\left(z_{j}^{(p)} / b_{j}^{(p)}=-1\right)}=\frac{4}{\sigma_{a}^{2}} z_{j}^{(p)} .
$$

Thus, these LLRs can be modeled as independent and identically distributed random variables with the conditional pdf $N\left(\frac{4 b_{j}^{(p)}}{\sigma_{a}^{2}}\right.$, $\left.\frac{8}{\sigma_{a}^{2}}\right)$.

Proposition 1 We consider the case where the receiver has side information (from a genie) that one of the two sequences $\underline{x}_{m}$ or $\underline{\widehat{x}}_{m}$ of length $m$ was transmitted. When the genie aided equalizer is provided with a priori information, the pairwise error probability that it chooses $\underline{\underline{x}}_{m}$ instead of $\underline{x}_{m}$ is:

$$
P_{\underline{x}_{m}, \underline{\widehat{x}}_{m}}=Q\left(\frac{\sqrt{\left\|\underline{\varepsilon}_{m}\right\|^{2}+4 m \mu^{2}}}{2 \sigma}\right) \text {. }
$$

where $\underline{\varepsilon}_{m}=H_{m} \underline{e}_{m}, \underline{e}_{m}$ is the error vector and $\mu=\frac{\sigma}{\sigma_{a}}$.

The proof of proposition 1 is given in the Appendix.

Let $E_{\underline{e}_{m}}$ be the set of all $\underline{e}_{m}$ achieving the minimum value $\min _{\underline{e}_{m}} \sqrt{\left\|\underline{\varepsilon}_{m}\right\|^{2}+4 m \mu^{2}}, \pi_{\underline{e}_{m}}$ be the probability that the input sequence $\underline{x}_{m}$ will be such that $\underline{\widehat{x}}_{m}=\underline{x}_{m}+\underline{e}_{m}$ is an allowable input sequence for some $\underline{e}_{m}$ in $E_{\underline{e}_{m}}$. Then the probability that the genie aided equalizer chooses an allowable sequence $\underline{\widehat{x}}_{m}$ instead of $\underline{x}_{m}$ is $\pi_{\underline{e}_{m}} Q\left(\frac{\min _{m} \sqrt{\left\|\underline{\varepsilon}_{m}\right\|^{2}+4 m \mu^{2}}}{2 \sigma}\right)$.

Corollary 2 The overall probability of error at the output of the MAP equalizer without genie, using the a priori information is lower bounded:

$$
P_{e} \geq l_{\underline{e}_{m}} \pi_{\underline{e}_{m}} Q\left(\frac{\min _{\underline{e}_{m}} \sqrt{\left\|\underline{\varepsilon}_{m}\right\|^{2}+4 m \mu^{2}}}{2 \sigma}\right),
$$

$l_{\underline{e}_{m}}$ is the length of the error sequence

Proof of corollary 2: For the MAP equalizer without genie, the probability of an error event $\xi_{m}$ of length $m$ is lower bounded by the probability achieved by the genie aided equalizer:

$$
P\left(\xi_{m}\right) \geq \pi_{\underline{e}_{m}} Q\left(\frac{\min _{m} \sqrt{\left\|\underline{\varepsilon}_{m}\right\|^{2}+4 m \mu^{2}}}{2 \sigma}\right) .
$$

Since $l_{\underline{e}_{m}}$ is the length of the error sequence, we deduce that the overall probability error is lower bounded as in (14). 
We will consider, in the following, different cases according to the value of $\mu$ to determine the expressions of the lower bounds on $P_{e}$ given in equation (14). For clarity of the presentation, we will focus on the case of MIMO systems with two transmit antennas and two receive antennas.

Corollary 3 In the case of unreliable a priori information, the overall probability of error is lower bounded:

$$
P_{e} \geq \frac{1}{2} * Q\left(\frac{\sqrt{d_{1}^{2}+16 \mu^{2}}}{2 \sigma}\right) .
$$

where $d_{1}=\min _{\underline{e}_{m}, m=4}\left\|\underline{\varepsilon}_{m}\right\|$.

Proof of corollary 3: Generally, when the a priori information at the input of the MAP equalizer are unreliable, errors occur in bursts. Thus, we do not consider isolated errors since they occur rarely and we obtain $m_{p} \geq 2$ for $p \in\{1,2\}$. Thus, the minimum value of $m=m_{1}+m_{2}$ is 4 . A lower bound on the error probability is then obtained by replacing $m$ by 4 and $\left\|\varepsilon_{m}\right\|$ by $d_{1}$. Hence, $l_{\underline{e}_{m}}=4$ and an exhaustive search by simulations shows that $\pi_{\underline{e}_{m}}$ is equal to $1 / 8$.

Corollary 4 When the a priori information are reliable, the overall probability of error is lower bounded:

$$
P_{e} \geq \frac{3}{2} * Q\left(\frac{\sqrt{d_{2}^{2}+8 \mu^{2}}}{2 \sigma}\right) .
$$

where $d_{2}=\min _{\underline{e}_{m}, m=2}\left\|\underline{\varepsilon}_{m}\right\|$.

Proof of corollary 4: In the case of reliable a priori information, a priori observations have more influence on the detection than channel observations. Since the a priori information are independent, the errors will not occur in bursts. Then, a lower bound on the error probability is obtained for isolated errors by replacing $m=m_{1}+m_{2}$ by 2 and $\left\|\underline{\varepsilon}_{m}\right\|$ by $d_{2}$. Hence, $l_{\underline{e}_{m}}=2$ and an exhaustive search by simulations shows that $\pi_{e_{m}}$ is equal to $3 / 4$. Let

$$
\mu_{\text {lim }}=\sqrt{\frac{d_{2}^{2}-d_{1}^{2}}{8}}
$$

when $d_{2}>d_{1}$.

We can easily show that when $\mu \leq \mu_{\text {lim }}$ (unreliable a priori information), $\min _{\underline{e}_{m}} \sqrt{\left\|\underline{\varepsilon}_{m}\right\|^{2}+4 m \mu^{2}}$ is reached for $m=4$ and $\left\|\underline{\varepsilon}_{m}\right\|=d_{1}$ and when $\mu \geq \mu_{\text {lim }}$ (reliable a priori information), $\min _{e_{m}} \sqrt{\left\|\underline{\varepsilon}_{m}\right\|^{2}+4 m \mu^{2}}$ is reached for $m=2$ and $\left\|\underline{\varepsilon}_{m}\right\|=d_{2}$. When $d_{2}<d_{1}$, the minimum value is reached for $m=2$ and $\left\|\underline{\varepsilon}_{m}\right\|=d_{2}$ for all $\mu$.

\section{SIMULATION RESULTS}

In this section, we propose to give simulation results to validate the analytical study.

We use a MIMO system with two transmit antennas and two receive antennas. The impulse responses of the channels between the transmit and receive antennas are: $h^{(1,1)}=(0.5,0.71,0.5)$, $h^{(1,2)}=(0.9,0.5,0.29), h^{(2,1)}=(0.29,0.9,0.5)$ and $h^{(2,2)}=$ $(0.5,0.6,0.7)$. The modulation used is the QPSK. We provide the MAP equalizer with a priori information on the transmitted bits $b_{j}^{(p)}$ modeled as independent and identically distributed random variables with the conditional pdf $N\left(\frac{4 b_{j}^{(p)}}{\sigma_{a}^{2}}, \frac{8}{\sigma_{a}^{2}}\right)$. For the considered MIMO channel, we have $d_{1}^{2}=2.7232$ and $d_{2}^{2}=1.4862$. Then, since $d_{1}>d_{2}$, we use the expression given in corollary 3 for all values of $\mu$, because it gives the minimum value of $\sqrt{\left\|\underline{\varepsilon}_{m}\right\|^{2}+4 m \mu^{2}}$.

On figure 1 , we plot the BER with respect to the signal to noise ratio (SNR), for different values of $\mu=\frac{\sigma}{\sigma_{a}}$. Each curve is obtained while the ratio $\mu$ is kept constant. The solid lines indicate the equalizer performance given by simulations. The dotted lines indicate the theoretical lower bounds calculated in the previous section. We notice that the theoretical curves approximate well the BER. This approximation becomes better at high SNR (for low values of BER).

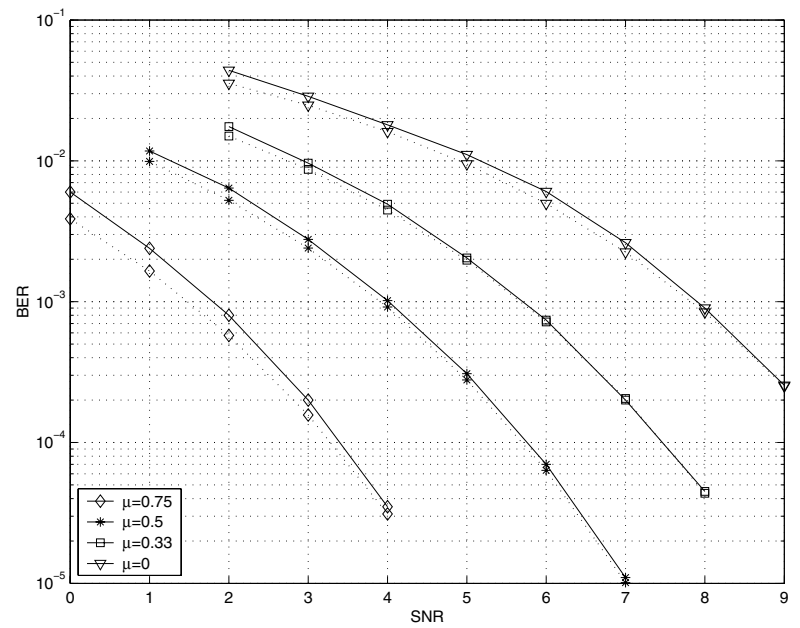

Fig. 1. BER versus SNR: comparison of the equalizer performance (solid curves) and the theoretical performance (dotted curves).

\section{CONCLUSION}

In this paper, we consider a transmission of QPSK symbols over a frequency selective MIMO system. We propose to study analytically, in this case, the impact of a priori information on the MAP equalizer performance. We give expressions of tight lower bounds on the error probability. Simulation results show that the analytical expressions give a good approximation of the equalizer performance. This work is a first step in the convergence analysis of iterative receivers with MAP equalization for MIMO systems.

\section{APPENDIX: PROOF FOR PROPOSITION 1}

We recall that the output of the MIMO channel during a burst is the $N T \times 1$ vector $\underline{y}=\left(y_{0}^{(1)}, \ldots, y_{T-1}^{(1)}, y_{0}^{(2)}, \ldots, y_{T-1}^{(2)}, \ldots, y_{0}^{(N)}\right.$, $\left.\ldots, y_{T-1}^{(N)}\right)^{H}$ defined as:

$$
\underline{y}=H \underline{x}+\underline{q},
$$

where $\quad \underline{x}=\left(x_{1-L}^{(1)}, \ldots, x_{T-1}^{(1)}, x_{1-L}^{(2)}, \ldots, x_{T-1}^{(2)}, \ldots, x_{1-L}^{(P)}, \ldots\right.$, $\left.x_{T-1}^{(P)}\right)^{H}$ is the $P(T+L-1) \times 1$ vector of transmitted symbols, $\underline{q}=\left(q_{0}^{(1)}, \ldots, q_{T-1}^{(1)}, q_{0}^{(2)}, \ldots, q_{T-1}^{(2)}, \ldots, q_{0}^{(N)}, \ldots q_{T-1}^{(N)}\right)^{H}$ is the complex Gaussian noise vector and $H$ is the $N T \times$ $P(T+L-1)$ channel matrix given by (3).

In addition, using (10) and (11), the a priori observations on symbols $x_{k}^{(p)}$ are given by :

$$
c_{k}^{(p)}=x_{k}^{(p)}+g_{k}^{(p)},
$$

where $g_{k}^{(p)}=\left(w_{2 k}+j w_{2 k+1}\right) \sim N_{\mathbb{C}}\left(0, \sigma_{a}^{2}\right)$, for $1-L \leq k \leq$ $T-1$ and $1 \leq p \leq P$.

Taking into account the a priori information, the a posteriori probability of the sequence $\underline{x}$ is given by:

$$
P(\underline{x} \mid \underline{y}, \underline{c}) \propto \exp \left(-\frac{\|\underline{y}-H \underline{x}\|^{2}}{\sigma^{2}}\right) \exp \left(-\frac{\|\underline{c}-\underline{x}\|^{2}}{\sigma_{a}^{2}}\right),
$$


where $\underline{c}=\left(c_{1-L}^{(1)}, \ldots, c_{T-1}^{(1)}, c_{1-L}^{(2)}, \ldots, c_{T-1}^{(2)}, \ldots, c_{1-L}^{(P)}, \ldots, c_{T-1}^{(P)}\right)^{H}$ is the $P(T+L-1) \times 1$ vector of a priori observations on the symbols. The data estimate according to the MAP sequence criterion is then given by:

$$
\underline{\widehat{x}}=\arg \min _{\underline{u}}\left(\|\underline{y}-H \underline{u}\|^{2}+\frac{\sigma^{2}}{\sigma_{a}^{2}}\|\underline{c}-\underline{u}\|^{2}: \underline{u} \in A^{P(T+L-1)}\right) .
$$

As in the case of no a priori information, we consider an error event $\xi_{m}$ of length $m$. This error event is that $\widehat{x}_{m}$ is better than $\underline{x}_{m}$ in the sense of the MAP sequence metric:

$$
\begin{gathered}
\xi_{m}:\left\|\underline{y}_{m}-H_{m} \underline{\widehat{x}}_{m}\right\|^{2}+\frac{\sigma^{2}}{\sigma_{a}^{2}}\left\|\underline{c}_{m}-\underline{\widehat{x}}_{m}\right\|^{2} \leq \\
\left\|\underline{y}_{m}-H_{m} \underline{x}_{m}\right\|^{2}+\frac{\sigma^{2}}{\sigma_{a}^{2}}\left\|\underline{c}_{m}-\underline{x}_{m}\right\|^{2},
\end{gathered}
$$

where $m=\sum_{p=1}^{P} m_{p}, \underline{y}_{m}, H_{m}$ and $\underline{c}_{m}$ are respectively the subvector of $y$, the subblock of $H$ and the subvector of $\underline{c}$ corresponding to the error interval.

Let $\underline{r}=\left(\underline{y}^{H}, \mu \underline{c}^{H}\right)^{H}, M=\left(H^{H}, \mu I_{P(T+L-1)}\right)^{H}$ and $\underline{s}=$ $\left(\underline{q}^{H}, \mu \underline{g}^{H}\right)^{H}$, with $\underline{g}=\left(g_{1-L}^{(1)}, . ., g_{T-1}^{(1)}, g_{1-L}^{(2)}, . ., g_{T-1}^{(2)}, \ldots\right.$. , $\left.g_{1-L}^{(P)}, . ., g_{T-1}^{(P)}\right)^{H}$. Using (19) and (20), we can write:

$$
\underline{r}=M \underline{x}+\underline{s} .
$$

Then, the data estimate according to the MAP sequence criterion is given by:

$$
\underline{\widehat{x}}=\arg \min _{\underline{u}}\left(\|\underline{r}-M \underline{u}\|^{2}: \underline{u} \in A^{P(T+L-1)}\right) .
$$

Hence, (23) is equivalent to:

$$
\xi_{m}:\left\|\underline{r}_{m}-M_{m} \underline{\widehat{x}}_{m}\right\|^{2} \leq\left\|\underline{r}_{m}-M_{m} \underline{x}_{m}\right\|^{2},
$$

$\underline{r}_{m}$ is the $(m+(m+P(L-1))) \times 1$ subvector of $\underline{r}$ and $M_{m}=$ $\left(H_{m}^{H}, \mu I_{(m+P(L-1))}\right)^{H}$ is the subblock of the matrix $M$ corresponding to the error interval.
We consider the case where the receiver has side information (from a genie) that one of the two sequences $\underline{x}_{m}$ or $\underline{\widehat{x}}_{m}$ was transmitted. When the genie aided equalizer is provided with $a$ priori information, the pairwise error probability that it chooses $\underline{\widehat{x}}_{m}$ instead of $\underline{x}_{m}$ is given by:

$$
P_{\underline{x}_{m}, \underline{\widehat{x}}_{m}}=Q\left(\frac{\left\|\underline{d}_{m}\right\|}{2 \sigma}\right),
$$

where $\underline{d}_{m}=M_{m} \underline{e}_{m}$. We can write:

$$
\left\|\underline{d}_{m}\right\|^{2}=\left\|\underline{\varepsilon}_{m}\right\|^{2}+\left\|\mu I_{(m+P(L-1))} \underline{e}_{m}\right\|^{2},
$$

where $\underline{\varepsilon}_{m}=H_{m} \underline{e}_{m}, \underline{e}_{m}$ is the error vector having $m$ first components equal to \pm 2 or $\pm 2 j$ and the others are equal to zero. Then, using (27) and (28), we obtain:

$$
P_{\underline{x}_{m}, \underline{\widehat{x}}_{m}}=Q\left(\frac{\sqrt{\left\|\underline{\varepsilon}_{m}\right\|^{2}+4 m \mu^{2}}}{2 \sigma}\right) .
$$

\section{REFERENCES}

[1] Douillard C., Jezequel M., Berrou C., Picart A., Didier P., Glavieux A., "Iterative correction of intersymbol interference: Turbo-equalization", Europ. Trans. On Telecomm., vol. 6, pp. 507-511, 1995.

[2] Sellami N., Roumy A., Fijalkow I., "The impact of both $a$ priori information and channel estimation errors on the MAP equalizer performance", IEEE transactions on signal processing, vol. 54, No 7, pp. 2716-2724, July 2006.

[3] G.D.Forney, Jr., "Lower bounds on error probability in the presence of large intersymbol interference ", IEEE Transactions on Communications, pp. 76-77, February 1972.

[4] Bahl L.R., Cocke J., Jelinek F., Raviv J., "Optimal decoding of linear codes for minimizing symbol error rate", IEEE Trans. Inf. Theory, vol.IT-32, pp. 284-287, March 1974.

[5] Benedetto S., Biglieri E., Principles of digital transmission with wireless applications, kluwer/Plenum, New York 1999.

[6] Ten Brink S., "Convergence of iterative decoding", IEEE Electronic Letters, vol. 35, pp.806-808, May 1999. 